\title{
Self-Organized Patchiness and Catastrophic Shifts in Ecosystems
}

\author{
Max Rietkerk, ${ }^{1 *}$ Stefan C. Dekker, ${ }^{1}$ Peter C. de Ruiter, ${ }^{1}$ Johan van de Koppel ${ }^{2}$
}

Unexpected sudden catastrophic shifts may occur in ecosystems, with concomitant losses or gains of ecological and economic resources. Such shifts have been theoretically attributed to positive feedback and bistability of ecosystem states. However, verifications and predictive power with respect to catastrophic responses to a changing environment are lacking for spatially extensive ecosystems. This situation impedes management and recovery strategies for such ecosystems. Here, we review recent studies on various ecosystems that link self-organized patchiness to catastrophic shifts between ecosystem states.

$\mathbf{E}$ cosystems are exposed to changes in climate, nutrient loading, or biotic exploitation. How ecosystems undergo such environmental changes on different scales of space and time is one of the main frontiers in ecology. Although environmental change can be slow and gradual, it may lead to sudden catastrophic change in the structure and functioning of ecosystems (1). Such catastrophes are commonly attributed to the existence of two alternative stable states in ecosystems (2), meaning that the dynamics of these systems are determined by two attracting states. Here, we define this as bistability.

${ }^{1}$ Department of Environmental Sciences, Copernicus Institute, Utrecht University, P.O. Box 80115, 3508 TC Utrecht, Netherlands. 'Spatial Ecology Department, Netherlands Institute of Ecology, P.O. Box 140, 4400 AC Yerseke, Netherlands.

*To whom correspondence should be addressed. E-mail: m.rietkerk@geog.uu.nl
Positive feedback control between consumers (e.g., plants) and limiting resources (e.g., water, nutrients) is considered to be the principle underlying catastrophic ecosystem shifts $(1-3)$. This picture emerged from models that ignore spatial interactions. Hence, these so-called mean field models predict bistability in ecosystems as a consequence of positive feedback. We interpret this as local bistability. Such mean field analysis is particularly helpful for understanding catastrophic shifts in homogeneous or well-mixed ecosystems. The sudden loss of transparency in shallow lakes provides an illustrative example $(4,5)$. However, verifications and predictive power of catastrophic shifts are lacking for spatially extensive, heterogeneous ecosystems. As a result, sustainable management and recovery strategies for such ecosystems have been difficult to devise; they require an understanding of the relation between feedback and spatial scale.
We review recent ecosystem studies that include feedback control and spatial scale (Table 1 and Fig. 1). These studies link feedback control to self-organized patchiness of consumers and resources, and they show that a resource concentration mechanism invoked by consumers explains the diversity of spatial structures in these ecosystems. Such consumers have previously been called "ecosystem engineers" (6). Spatial self-organization is not imposed on any system but emerges from fine-scale interactions owing to internal causes (7). Moreover, model outcomes show that ecosystems where this resource concentration mechanism operates exhibit bistability between a specific spatially structured and homogeneous ecosystem state. We define the bistability at large spatial scales predicted by these spatially explicit models as global bistability.

Similar to the mean field models, global bistability in spatially explicit models is associated with catastrophic shifts at large spatial scales between coexisting stable states. Hence, these results stress the importance of self-organized patchiness for a better understanding of catastrophic shifts. Increased resource scarcity leads to spatial reorganization of consumers and resources in these model ecosystems, and an ecosystem state develops with localized structures observed in reality. Once resource scarcity reaches a threshold, the system shifts toward a homogeneous state

Table 1. Overview of references describing self-organized patchiness in some major ecosystems and the mechanisms involved.

Ecosystem References Pattern characteristics (scale) Mechanisms involved

Arid $\quad(8,11) \quad$ Spots, labyrinths, gaps $(1 \mathrm{~m})$ and stripes $(10 \mathrm{~m})$
(Fig. 1C)

$(9,14) \quad$ Periodic spots and bands (10 to $100 \mathrm{~m})$

(12) Spots, labyrinths, gaps, and stripes (10 to $100 \mathrm{~m}$ ) (Fig. 1, A and B)

(13) Disordered spots and clustered spots on hillslope contours (10 to $100 \mathrm{~m}$ )

Savanna

Peatland

Isolated spots of trees and shrubs in grass matrix

(10 to $100 \mathrm{~m})$ (Fig. 1, D and E)

String patterns $(10 \mathrm{~m})$
Maze and string patterns perpendicular to flow direction (10 m) (Fig. 1, F and G)
Redistribution of soil water due to positive feedback among plant biomass, extent of root system, and water uptake

Short-range facilitation and long-range competition for limiting water

Redistribution of surface water due to positive feedback between plant cover and water infiltration

Competition for limiting water

Short-range facilitation and long-range competition for limiting nutrients

Ponding of surface water upstream from hummocks combined with positive feedback between hummock occurrence and water table depth

Convective transport of limited nutrients in the groundwater toward areas with higher plant biomass, driven by differences in transpiration rate 
without consumers functioning as ecosystem engineers. Increasing resource availability does not recover these localized structures, because the resource concentration mechanism fails. This phenomenon is called hysteresis, meaning that specific spatial structures may develop in real ecosystems that only arise when resource availability is decreased, but not when increased. Therefore, we propose the hypothesis that imminent catastrophic shifts in ecosystems can be predicted by self-organized patchiness.

\section{Arid Ecosystems}

Self-organized patchiness and the resource concentration mechanisms involved have been reported from various ecosystems (Table 1 and Fig. 1), among which arid ecosystems are the most prominent $(8-14)$. The self-organized patchiness in these ecosystems differs in scale and shape. Patterns reported are gaps, labyrinths, stripes ("tiger bush") (Fig. 1, A to C), and spots ("leopard bush").

The general mechanism underlying this self-organized patchiness is a positive feedback between plant growth and availability of water. Higher vegetation density allows for higher water infiltration into the soil (because of root penetration) and lower soil evaporation (because of shading). As a result, vegetation persists once present, but bare soil is too hostile for recolonization after the vegetation disappears, implying that the present state of the vegetation depends on its history (3).

Recent studies link this positive feedback with subsequent redistribution of water resources $(10-12)$. Lateral flow of subsurface soil water at a scale of $0.1 \mathrm{~m}$, driven by differences in evapotranspiration, explains regular patterning of grasses in the Negev desert (11) (Fig. 1C). Redistribution of surface runoff water at a scale of $10 \mathrm{~m}$, driven by differences in water infiltration, elucidates the formation of self-organized patchiness in arid bushlands (12) (Fig. 1, A and B). These observations show that similar patterns of self-organized patchiness may emerge at different scales. Ecosystem transitions involve a sequence of emerging patterns of various forms induced by decreased rainfall. Vegetation states include homogeneous cover, gaps, labyrinths or stripes, and spots, in that order $(11,12,14)$. More important, in these models the vegetation shifts catastrophically from the spotted state to a bare homogeneous state if rainfall is decreased beyond a threshold. This can be attributed to global bistability of the spotted and bare states. Hence, a predictable form of self-organized patchiness may indicate imminent catastrophic shift to a bare homogeneous state. Increased rainfall may not recover the spotted state, because the resource concentration mecha- nism (concentration of soil water under vegetated patches) fails (11).

\section{Savanna Ecosystems}

In nutrient-poor Savanna ecosystems, periodic and aperiodic isolated spots of trees and shrubs
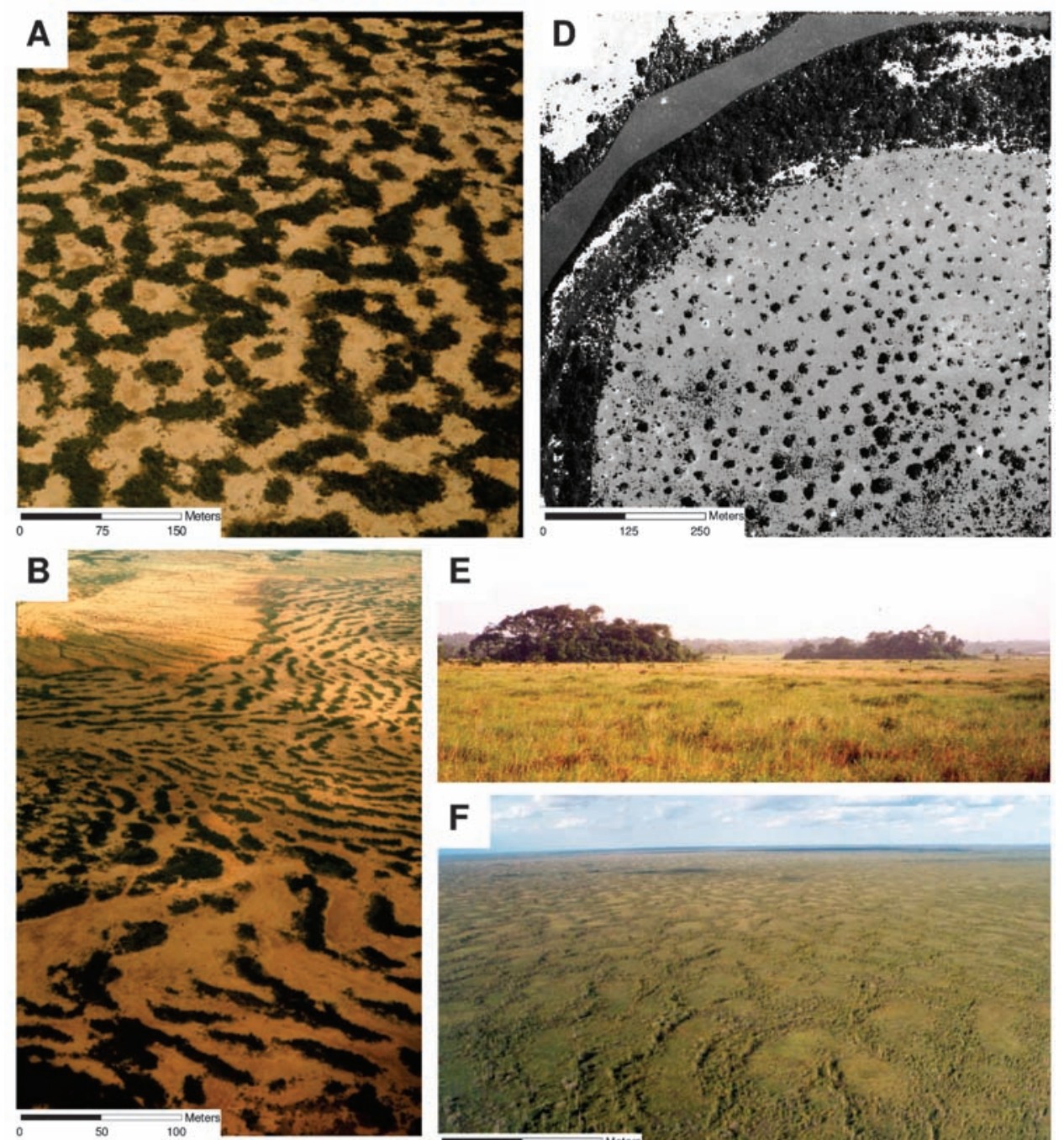

E
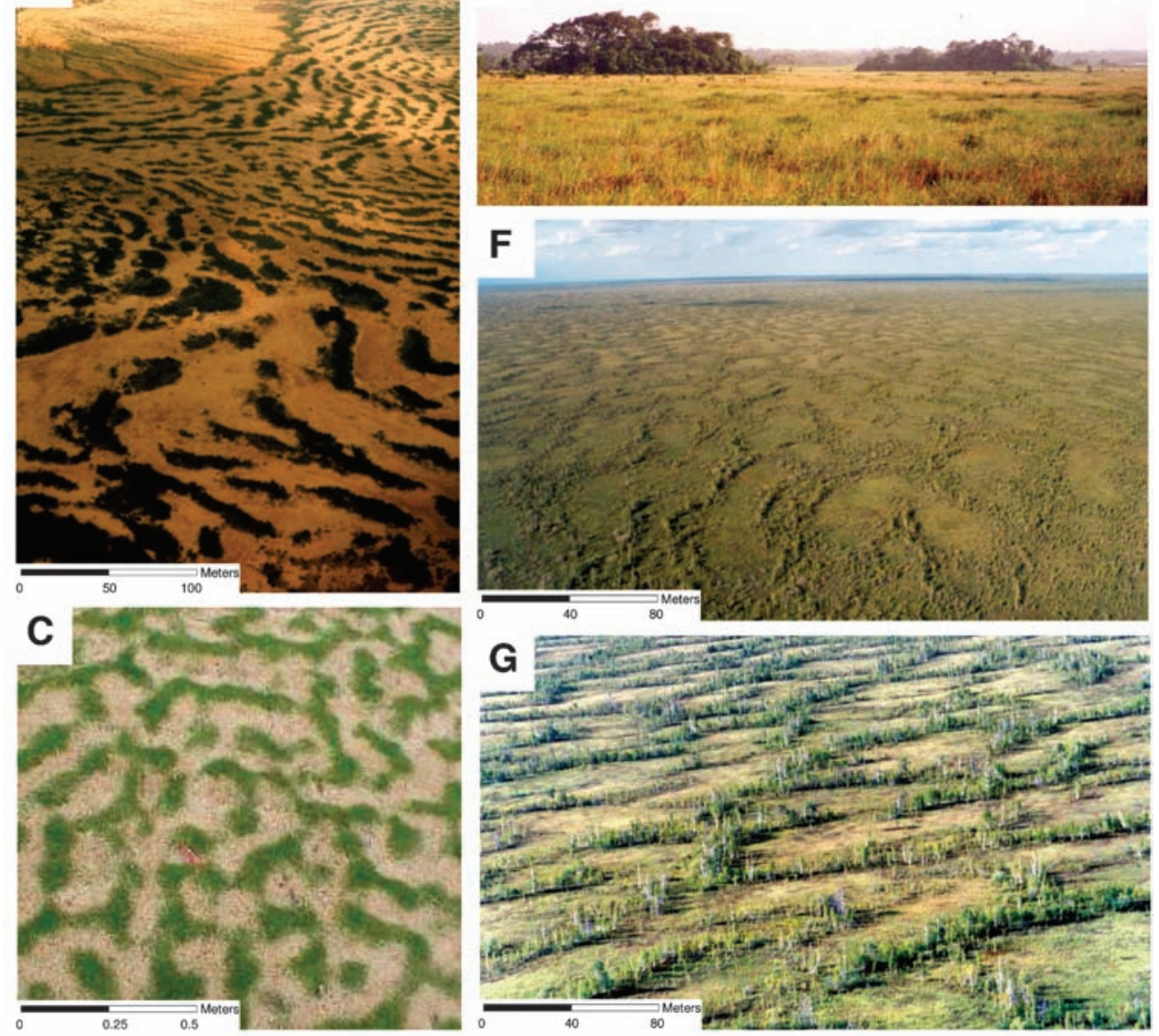

Fig. 1. Field observations. (A to C) Arid ecosystems: (A) Labyrinth of bushy vegetation in Niger [(12), (C) 2002 University of Chicago]; (B) Striped pattern of bushy vegetation in Niger; (C) Labyrinth of perennial grass Paspalum vaginatum in Israel [(11), (C) 2001 American Physical Society]. (D and E) Savanna ecosystems: Aerial and ground photographs of spots of tree patches in Ivory Coast and French Guiana, respectively [(15), (C) 2002 American Physical Society]. (F and G) Peatlands: Regular maze patterns of shrubs and trees in western Siberia [(25), (C) 2004 University of Chicago]. Scales of oblique aerial photographs [all panels except (E)] are order-of-magnitude approximations of distance in the $x$ direction shown in the scale bars. 


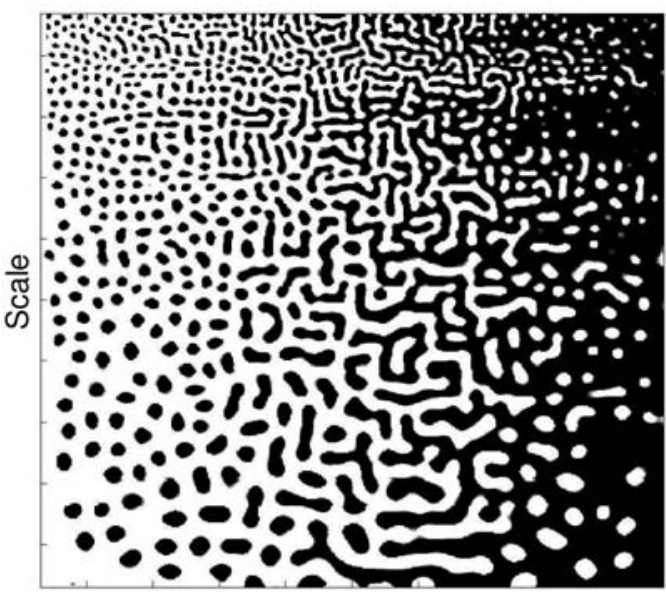

Strength

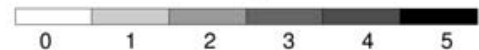

Fig. 2. Model results from a modified version of the Thiery et al. (30) cellular automaton, showing stable isotropic vegetation patterns after time step $t=15$ (supporting online text and movie S1). Grid size is $500 \times 500$ cells. Black areas represent vegetation in maximum state 5 ; white areas represent bare soil in minimum state 0 . Simulations were started by randomly introducing vegetation state 2 on $5 \%$ of the cells. Vegetation patterns are the result of fine-scale positive feedback and coarse-scale negative feedback On the $x$ axis, the strength of the positive feedback is increased; on the $y$ axis, the scale of influence is decreased. Patterns change from spots to labyrinths and gaps at different spatial scales.

at a scale of 10 to $100 \mathrm{~m}$ in grassland are observed (15) (Fig. 1, D and E). Lejeune et al. (15) proposed an interaction-redistribution model of vegetation dynamics generating these patterns even under homogeneous conditions. Their model is grounded on the balance between short-range facilitation and long-range competition. The mechanistic base is provided by observations that in nutrientpoor environments, trees and shrubs can have positive effects on each other and on themselves because of local nutrient accumulation as well as the ability of long superficial roots to track scarce nutrients from the surroundings (16-18).

Other plausible mechanisms coupling short-range facilitation with long-range competition are nutrient retention by the binding of soil by roots, raindrop interception, and runoff retention, all of which prevent erosion (3). This forms the mechanistic base of the concept of "islands of fertility" (19), explaining soil fertility under canopies of trees and shrubs by local nutrient accumulation and recycling. The observed patterns are interpreted as localized structures arising from global bistability between a "bare" state (no trees and shrubs) and a selforganized patchy state. Hence, Lejeune et al. (15) predicted the possibility of a stable homogeneous state and a self-organized patchy state for the same resource avail- ability, with catastrophic shifts between these states. As resource availability diminishes, the vegetation goes through a predictable sequence of emerging patterns comparable to that described above for arid ecosystems $(11,12,14,15)$.

\section{Peatland Ecosystems}

Bogs in North America and Eurasia commonly show various spatial patterning $(20,21)$, including regular string patterns of densely vegetated bands at a scale of $10 \mathrm{~m}$ (hummocks forming ridges) oriented perpendicular to the slope, alternating with more sparsely vegetated wetter zones (hollows forming pools) (22-24). Other regular patterns recently reported are maze patterns: bands densely vegetated by vascular plants at a scale of $10 \mathrm{~m}$ in a more sparsely vegetated matrix of predominantly nonvascular plants (25) (Fig. 1, F and G). A general mechanism underlying patterning in these ecosystems is a positive feedback between plant productivity and groundwater depth on elevated, drier sites, mainly due to increased production of vascular plants.

Rietkerk et al. (25) proposed the convective transport of nutrients in the groundwater toward areas with higher vascular plant biomass, driven by differences in transpiration, as a mechanism to explain regular string and maze patterns. If plant productivity is limited by nutrient flow, this local "nutrient accumulation" mechanism may lead to self-organized patchiness such as the observed string patterns on slopes and maze patterns on flat ground. Mean field models including this positive feedback allow for the occurrence of local bistability (thin peat with high water table coexisting with thick peat with low water table) for the same precipitation lev$\mathrm{el}$, and catastrophic shifts between these states $(26,27)$. Rietkerk et al. (25) predicted the possibility of global bistability (nonhomogeneous equilibrium with vascular plants and homogeneous equilibrium without vascular plants) for the same precipitation and nutrient input level, and catastrophic shifts between these states. Here, the vegetation also goes through a sequence of predictable patterns with decreasing nutrient input.

\section{Self-Organized Patchiness and Catastrophic Shifts}

Mean field models that ignore the relation between feedback and spatial scale predict local bistability and catastrophic shifts in the ecosystems discussed here $(1,3,26-28)$. Recent studies show that spatially explicit models including the resource concentration mechanism predict global bistability associated with catastrophic shifts at large spatial scales and self-organized patchiness. Observations confirm the model results. A variety of mechanisms in ecosystems lead to resource concentration through consumerresource feedback. The consumers harvest resources from their surroundings; in the ecosystems discussed here, harvest is facilitated by mass flow of resources toward consumers, triggered by the consumers themselves. Furthermore, consumers spread relatively slowly as compared to flow of resources. A general pattern emerging from these observations is that consumers are positively associated with resource abundance at short spatial range, but negatively at long spatial range (29). Thus, a common principle applies to these locally reinforced consumers, in that there is a positive feedback effect that is short-ranged and a negative feedback effect that is long-ranged. This is a necessary condition for selforganized patchiness to form (14).

We generated new results from a simple cellular automaton based on the model of Thiery et al. (30) exemplifying how such scale-dependent feedback can explain a diversity of patterns in ecosystems (Fig. 2, supporting online text, and movie S1). The differences in structure and scale of patchiness are the result only of varying strength and scale of feedback influence (14), illustrating the general nature of the underlying scale-dependent mechanisms explaining selforganized patchiness in ecosystems.

The notion of scale-dependent feedback controlled by the resource concentration mechanism is crucial for a predictive theory of catastrophic shifts in ecosystems. This suggests that catastrophic shifts can be predicted by self-organized patchiness (Fig. 3). Therefore, the concepts of catastrophic shifts and self-organized patchiness are tightly linked, whereby a scale-dependent feedback is triggered by resource concentration.

Ecosystems with scale-dependent feedback resemble the activator-inhibitor system first described by Turing (31). In the ecosystems discussed here, the inhibition effect results from the large-scale depletion of a resource that is consumed during the localized production of the activator (consumer). The activator effect results from the local positive feedback control between the consumer and its limited resource (water or nutrient availability). These ecosystems represent a class of activatorinhibitor system that has been recognized earlier as "activator-depleted substrate systems" (32). Indeed, theoretical analyses of an activator-depleted substrate system also predict global bistability and catastrophic shifts between spotted and uniform states (33). Experimental evidence of global bistability and 
hysteresis between states of spots and stripes comes from such a system as well $(34,35)$.

Most theoretical approaches to selforganized patchiness in ecosystems are based on the same framework of models as are used to explain pattern formation in chemical systems and biological pattern formation on sea shells (32) and animal coats (36). Here, we concentrated on the overlap between ecosystems exhibiting both selforganized patchiness and catastrophic shifts due to global bistability. The resource concentration mechanism invoked by ecosystem engineers provides a general explanation, because ecosystem engineers at low densities may be unable to harvest resources from the surroundings. We suggest that all ecosystems with selforganized patchiness resulting from a resource concentration mechanism will also exhibit catastrophic shifts.

\section{Challenges Ahead}

Linking self-organized patchiness with catastrophic shifts by the resource concentration mechanism may help to bridge the present gaps among theory, observation, and management (2). The link may be crucial to a predictive theory of catastrophic shifts from which early-warning systems can be developed on the basis of spatial explicit timeseries data. This is because predictable forms of selforganized patchiness may

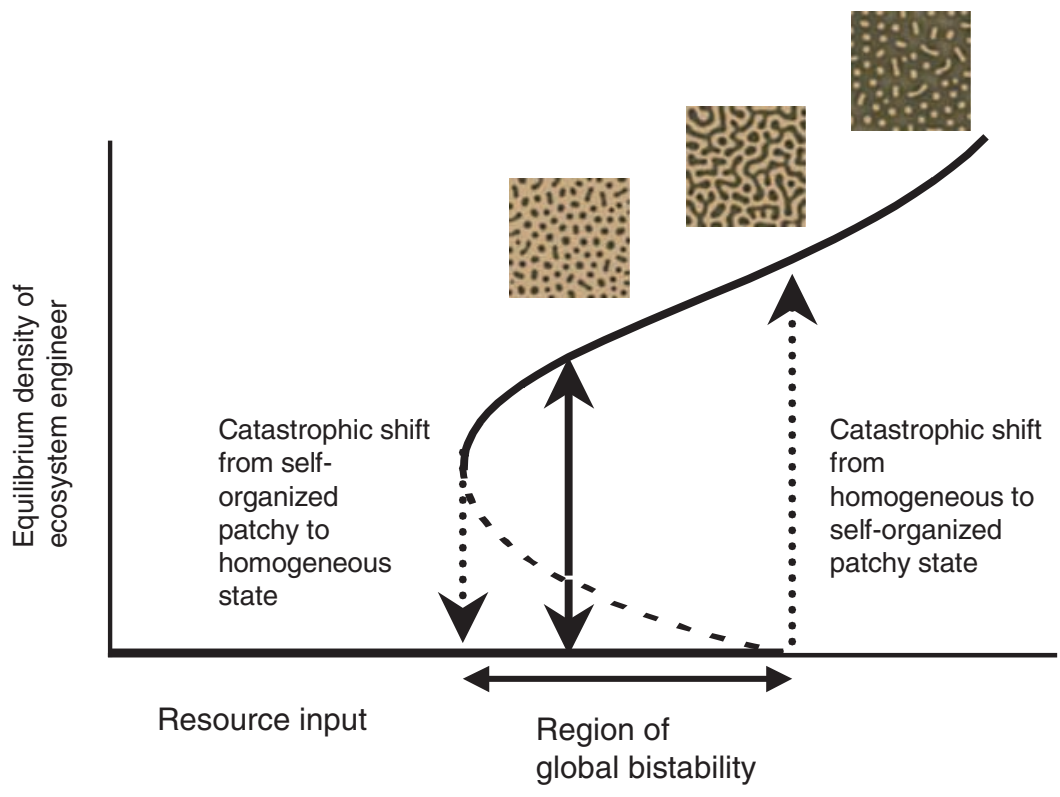

Fig. 3. Model showing how ecosystems may undergo a predictable sequence of emerging self-organized patchiness as resource input decreases or increases $(11,12,14$, 25). Thick solid lines represent mean equilibrium densities of consumers functioning as ecosystem engineers. Dotted arrows represent catastrophic shifts between selforganized patchy and homogeneous states, and vice versa. Dark colors in the insets represent high density. The range of resource input for which global bistability and hysteresis exists is between these dotted arrows. Solid arrows represent development of the system toward the coexisting self-organized patchy state or homogeneous state, depending on initial ecosystem engineer densities. indicate imminent cat-

astrophic shifts if resource input decreases in time (Fig. 3). For instance, the spotted state may develop only when resource input is decreased, not when it is increased. This means that a snapshot in time of a spotted state would already indicate imminent catastrophic shift.

Human management strategies could be directed toward preserving and restoring self-organized patchiness and its natural resource concentration function $(12,37)$. Vegetation structures in resource-poor agroecosystems, such as the African Sahel, may lose this function because of overgrazing by cattle, leading to catastrophic shifts to a ences, seasonality in rainfall, and random processes affect self-organized patchiness and ecosystem resistance against catastrophic shifts. Furthermore, the fact that selforganized patchiness and catastrophic shifts may occur at different spatial and temporal scales $(11,12)$ provides new perspectives for fine-scale and relatively short-term experiments to predict large-scale self-organized patchiness and catastrophic shifts in ecosystems.

\section{References and Notes}

1. M. Scheffer et al., Nature 413, 591 (2001).

2. M. Scheffer, S. R. Carpenter, Trends Ecol. Evol. 18, 648 (2003).
3. M. Rietkerk, J. van de Koppel, Oikos 79, 69 (1997).

4. M. Scheffer et al., Trends Ecol. Evol. 8, 275 (1993).

5. S. R. Carpenter et al., Ecol. Appl. 9, 751 (1999).

6. C. G. Jones et al., Oikos 69, 373 (1994).

7. P. Rohani et al., Trends Ecol. Evol. 12, 70 (1997).

8. C. Klausmeier, Science 284, 1826 (1999).

9. P. Couteron, O. Lejeune, J. Ecol. 89, 616 (2001).

. R. HilleRisLambers et al., Ecology 82, 50 (2001).

1. J. Von Hardenberg et al., Phys. Rev. Lett. 87, 198101 (2001).

M. Rietkerk et al., Am. Nat. 160, 524 (2002).

N. M. Shnerb et al., Phys. Rev. Lett. 90, 038101 (2003).

\section{(2004).}

15. O. Lejeune et al., Phys. Rev. E 66, 010901 (2002).

16. A. J. Belsky, Ecology 75, 922 (1994).

17. R. M. Callaway et al., Nature 417, 844 (2002).

18. J. F. Bruno et al., Trends Ecol. Evol. 18, 119 (2003).

19. W. H. Schlesinger et al., Ecology 77, 364 (1996).

20. R. A. Lindsay et al., Aquilo Ser. Bot. 21, 69 (1985).

21. L. R. Belyea, J. Lancaster, J. Ecol. 90, 223 (2002).

22. Y. Sakaguchi, Bull. Dept. Geogr. Univ. Tokyo 12, 35 (1980).

23. D. R. Foster et al., Nature 306, 256 (1983).

24. D. K. Swanson, D. F. Grigal, Oikos 53, 509 (1988).

25. M. Rietkerk et al., Am. Nat. 163, 699 (2004).

26. D. W. Hilbert et al., J. Ecol. 88, 230 (2000).

27. L. R. Belyea, R. S. Clymo, Proc. R. Soc. London Ser. B 268, 1315 (2001).

28. M. Rietkerk et al., Oikos 80, 241 (1997).

29. M. Rietkerk et al., Plant Ecol. 148, 207 (2000).

30. J. M. Thiery et al., J. Ecol. 83, 497 (1995).

31. A. Turing, Philos. Trans. $R$. Soc. London Ser. B 273, 37 (1952).

32. H. Meinhardt, The Algorithmic Beauty of Sea Shells (Springer, Berlin, 1995).

33. S. L. Judd et al., Physica D 136, 45 (2000)

34. V. Castets et al., Phys. Rev. Lett. 64, 2953 (1990).

35. Q. Ouyang et al., J. Phys. Chem. 96, 6773 (1992).

36. J. D. Murray, Mathematical

Biology, vol. 19 of Biomathematics Texts (SpringerVerlag, Berlin, 1989).

37. J. Ludwig et al., Landscape Ecology, Function and Management: Principles from Australia's Rangelands (CSIRO, Collingwood, Victoria, Australia, 1997).

38. We thank W. Bleuten, P. Couteron, C. Klausmeier, E. Lapshina, O. Lejeune, E. Meron, and M. Wassen for sharing photographs, and M. Scheffer, P. Herman, and two anonymous referees for thoughtful comments that increased the clarity of the manuscript. Supported by a VIDI grant of the Netherlands Organization of Scientific Research (NWO-ALW) (M.R.).

Supporting Online Material

www.sciencemag.org/cgi/content/full/305/5692/1926/ DC1

SOM Text

Movie S1 